\title{
Causes of Schedule Overruns in Pakistani Construction Industry
}

\author{
${ }^{1}$ Dr. Tahir Nawaz, ${ }^{2}$ Amjad Ali Ikram, and ${ }^{3}$ Aftab Ahmed Qureshi \\ Associate Professor, Center for Advanced Studies in Engineering, Islamabad, Pakistan. \\ MSc Engineering Management, Center for Advanced Studies in Engineering, Islamabad, Pakistan. \\ MSc Engineering Management, Center for Advanced Studies in Engineering, Islamabad, Pakistan.
}

\begin{abstract}
In the modern construction industry sector there are multiple stakeholders which affect on the time performance of the projects. These have been grouped into five categories which are; the government, consultants, contractors, the clients and other factors. This paper is an attempt to identify and analyze the causes of schedule overruns by targeting the construction industry professionals. In addition to this, pertinent literature on the subject has been studied and made part of this research work. A survey questionnaire was distributed among respondents on time performance of various completed and ongoing construction projects.

The major findings and conclusions derived from this paper are; paucity of funds, , rapid market fluctuations, hegemony of market players, ineffectiveness of site management, delay in site mobilization, lack of the modern design software, delay in approvals of shop drawings, frequent changes during execution, lack of involvement of client in planning. These issues can be addressed through adequate supply of funds, quick decisions making, good planning, market stability, site management and timely mobilization and use of Project Management Information System.
\end{abstract}

Keywords: Causes, Construction, Pakistan, Prime Stakeholders,. Schedule Overruns.

\section{INTRODUCTION}

It is quite unfortunate that majority of construction projects fail to meet the schedule baselines. This phenomenon is equally applicable to all levels of projects i.e.; from major, capital, mega or giga projects. Due to this unhealthy trend, the industry has virtually become infamous across the globe. It is also worth mentioning here that, construction industry potential has never been fully exploited despite the fact that this sector is the major contributor in Gross Domestic Product of a country.

1.1 Construction Industry acquires diversified range of stakeholders like; design professionals, architects, engineers, contractors, sub contractors, suppliers and owners. Similarly it is also directly or indirectly affected by external factors and actors like government regulatory agencies, environmental agencies, law and order, market hegemonic forces, force majeure, lack of accessibility to the site and poor soil conditions. These all internal and external factors and actors play a substantial role towards time performance of construction projects. According to Haseeb (2011), there is an ongoing debate over this retrogressive phenomenon with a purpose to eliminate, deflect or mitigate their effects and occurrence. Project Management Institute's famous Project Management Body of knowledge (PMBOK-4th Edition) advocates the philosophy of Project Integration Management. The basic aim for Project Integration Management is to synchronize and harness, all the subsidiary plans in order to achieve project goals on time and within budget. Unfortunately this aspect is neglected or forgotten, as a result of which projects are subjected to or confronted with massive constructability issues, conflicts and clashes, design errors, frequent change orders, cost overruns and late completions. In the worst scenarios, some projects are completely abandoned when contractor disserts out of frustration from the jobsite.

1.2 Lu (2001) says that planning is a very significant phase in which all stakeholders have a significant role to play. Lack of adequate planning results into slippage of schedule baselines, claim for Extension of Time (EOT), change orders and extra costs .Meanwhile Han(2009) considers site management itself an important issue which warrants diligent consideration. Inefficient site management causes schedule slips and construction wastages.

1.3 In this paper a substantial effort has been made to identify, the most probable causes of schedule overruns in order to find out best ways for addressing these issues.

\section{SCOPE AND OBJECTIVES}

To identify the causes of schedule overruns in the construction projects and recommend viable recommendations in order to address these issues properly. 


\section{LITERATURE REVIEW}

3.1 Construction industry is well known for its wide employment opportunities and a significant contributions for the development and economic growth of any country. Haseeb(2011) investigates that, one of the major challenges which this industry facing is, slippage of projects from their schedule baselines. This phenomenon taxes the sponsors or clients more heavily due to poor productivity and loss of revenue .According to $\mathrm{Lu}(2001)$, the majority of schedule delays or dips arise out of client's actions; such as poor financial arrangement, slow in decision making, and lack of intimate involvement in planning .Moreover Han(2009) considers the lack of state-of-the-art methodology and technology as well as modern tools and techniques impede the progress of the project drastically. It also entails reworks, scrap, change orders, design errors and constructability issues. Out of Triple Constraints of Time, Cost and Scope; performance of time and cost for a construction project are directly linked together. Slip in schedule baseline will directly influence cost overruns. Apolot(2011) advocates that normally there are five major causes of schedule delays which are; scope revisions, delays in progress payments, exchange rate fluctuations, poor site management and dearth of resources.

3.2 Sambavisan (2007) also suggests that lack of communication among primary stakeholders, results into conflicts with regard to project methodologies, designs, integration of subsidiary management plans, and constructability issues during execution phase. When such environment prevails, then design engineers, architects and the contractors cannot synchronize their efforts for the common project goals. This problem and situation gets compounded when the designers or architects are egoistic and rigid in their approach towards dealing with contractors. They try to enforce their own verdict, may it be on the fault lines.

3.3 Hoai(2008) has very correctly highlighted that sometimes consultants become so rigid that either they do not give approvals of shop drawings or try to delay as much as they can. Instances have also been reported when consultants added additional scope in order to pad up project cost without formal approval of the client. That is why Vindali(2002) commented on this unprofessional attitude which have resulted into $29 \%$ of time overruns. Once the project is formally kicked off for execution of works, the contractor and consultant get involved in fire fighting because they had not spent enough time for detailed planning of the project. To overcome this issue Kagapaylan(2008) suggests that poor alignment of goals between contractors and the clients, compromises time performance of the projects.

3.4 FIFA World Cup Stadia 2010 project which was very meticulously planned and executed in South Africa, was not even exception to time overruns. In that project, Bayoli (2011) has identified the factors which affected this mega project. These factors arose out of actions of client; designers and the contractors. The main factors remained; inaccurate quantity surveying, change orders, design errors and omissions, slowness in decision making by client, lack of communication management, delay in work approval and incomplete drawings.

3.5 Now the question arises, how to bridge-up this gap so that projects can be completed on time. Ramathan(2012) and Ford(2008) developed three types of project controls i.e. pushing project teams to work faster, having staff work over time and adding up additional staff. This methodology relies upon resource management for increasing the productivity or reducing reworks. For the purpose of monitoring the schedule performance index (SPI), a proper system of feedback is tied with this system of Dynamics model. According to Shah(2004), construction industry is a competitive high-risk business in the world. Countries have adopted numerous project delivery systems and methods in order to averse the risks; such as fixed price or lump sum contract, design build, partnering and joint ventures. Malaysian construction industry has successfully reaped the benefits of partnering in construction projects. Through this arrangement, the industry achieved high customer satisfaction, enhanced communications, reduced reworks, superior quality, improved time control and lesser disputes.

3.6 Most of the reworks in construction projects arise out of schedule delays. Kasimo(2012) and Love(2004) suggest that this trend can only be eliminated or mitigated if client and design team, coordinate at each phase, manage the site effectively, improve project communications and adopt modern procurements technique like JIT in a holistic manner. Further to this, government should adopt conducive taxation and public procurement policy through which hegemony of market forces and price escalations can be controlled.

\section{Research Methodology}

4.1 This research was spanned over two phases i.e.; data collection and data analysis. For the purpose of data collection, a questionnaire was designed by incorporating the causes which slip the schedule baselines of projects. The questionnaire was divided into two parts. In part A, general information about respondents was 
asked so that reliability and validity of the data can be ensured. In part B, specific questions were asked. These questions were grouped into five domains, and each domain depicted a particular stakeholder of projects. These stakeholder were; the government, contractors, consultants, owners/clients and other factors. Each domain had one major question having ten options (causes). There were total of five major questions and fifty causes of schedule overruns.

4.2 First the authors discussed about the questionnaire, with industry related contractors, construction managers, architects, design professionals, suppliers, sub contractors and supervisors for ascertaining and confirming whether this questionnaire was enough for data collection. After through discussions with renowned and well reputed industry professionals like M/S Ahmad Zaka \& Associates, M/S Amin Tariq \& Associates, M/S Suhail Ahmad \& Associates, M/S Shami Associates, M/S Tijaarat Developers and M/S Adeel Associates, the questionnaire was approved with minor modifications and adjustments.

4.3 In the next step, the authors carried out random sampling for the selection of the respondents. A total of 150 questionnaires were distributed among the respondents from government departments, contractors, consultants, clients, professional civil engineers and project managers. Apart from this the authors interviewed the skilled and unskilled labor who are working on different construction projects. The panel of authors received 101 completed questionnaires, which comes to about 67.33 percent of response. Once this major milestone was achieved, the authors analyzed the data by using statpro software.

\section{Results and Discussions}

This research has been divided into five domains, the details of results are described in the succeeding paragraphs:-

5.1 Schedule Slippage Due to Government. Government has a concrete role to play for the construction industry. Its policies and regulations have direct bearing over the performance of the construction projects. If a market which is controlled and dominated by few big suppliers or manufacturers, it will exert devastating effects on construction industry; because they will monopolize and enforce their own selling prices and rates. This trend and practice best flourishes under fragile economic environments as it prevail today.

Weak economy is a hurdle towards timely completion of projects because of insufficient funds necessary for making progress payments and maintenance of cash flows. This problem is escalated exponentially when taxes are high for procurement of construction materials.

In developing economies, the involvement of politicians in the developmental works and infrastructure projects is enormous. They try to steer these projects according to their vested interests. Similarly political uncertainty adds fuel to this situation.

In the backdrop of these circumstances, though government has adopted stringent measures like establishment of Planning Commission of Pakistan and Public Procurement Regulatory Agency (PPRA) but still there is a lot of work which needs to be done. In this domain of questionnaire, the majority of respondents indicated top two causes i.e.; paucity of funds and delay in progress payments, with a average ratings of 1.45 and 2.71 respectively. The other causes along with ratings are described in table 1 and fig- $1(\mathrm{a} \& \mathrm{~b})$ below:

\begin{tabular}{|c|c|c|c|c|c|c|c|c|c|c|c|c|}
\hline & 1 & 2 & 3 & 4 & 5 & 6 & 7 & 8 & 9 & 10 & \begin{tabular}{|l|} 
Rating \\
Average
\end{tabular} & $\begin{array}{c}\text { Response } \\
\text { Count } \\
\end{array}$ \\
\hline $\begin{array}{l}\text { Paucity of } \\
\text { Funds }\end{array}$ & $\begin{array}{c}87.6 \% \\
(92)\end{array}$ & $\begin{array}{c}4.8 \% \\
(5)\end{array}$ & $\begin{array}{c}0.0 \% \\
(0)\end{array}$ & $\begin{array}{c}1.0 \% \\
(1)\end{array}$ & \begin{tabular}{|c|}
$3.8 \%$ \\
$(4)$
\end{tabular} & $\begin{array}{c}0.0 \% \\
(0)\end{array}$ & $\begin{array}{c}1.0 \% \\
(1)\end{array}$ & $\begin{array}{c}0.0 \% \\
(0)\end{array}$ & $\begin{array}{l}1.0 \% \\
(1)\end{array}$ & $\begin{array}{c}1.0 \% \\
(1)\end{array}$ & 1.45 & 105 \\
\hline $\begin{array}{l}\text { Lack of } \\
\text { control } \\
\text { overprices }\end{array}$ & $\begin{array}{c}3.8 \% \\
(4)\end{array}$ & \begin{tabular}{c|}
$1.0 \%$ \\
$(1)$
\end{tabular} & $\begin{array}{c}28.6 \% \\
(30)\end{array}$ & $\begin{array}{c}56.2 \% \\
(59)\end{array}$ & \begin{tabular}{|c|}
$2.9 \%$ \\
$(3)$
\end{tabular} & \begin{tabular}{|c|}
$2.9 \%$ \\
$(3)$
\end{tabular} & $\begin{array}{c}1.0 \% \\
(1)\end{array}$ & $\begin{array}{c}3.8 \% \\
(4)\end{array}$ & $\begin{array}{c}0.0 \% \\
(0)\end{array}$ & $\begin{array}{c}0.0 \% \\
(0)\end{array}$ & 3.85 & 105 \\
\hline $\begin{array}{l}\text { Fragile } \\
\text { Economy }\end{array}$ & $\begin{array}{c}0.0 \% \\
(0)\end{array}$ & \begin{tabular}{c|}
$1.0 \%$ \\
$(1)$
\end{tabular} & $\begin{array}{c}2.9 \% \\
(3)\end{array}$ & \begin{tabular}{|c|}
$4.8 \%$ \\
$(5)$
\end{tabular} & $\begin{array}{c}66.7 \% \\
(70)\end{array}$ & $\begin{array}{c}19.0 \% \\
(20)\end{array}$ & $\begin{array}{c}1.0 \% \\
(1)\end{array}$ & $\begin{array}{c}1.9 \% \\
(2)\end{array}$ & $\begin{array}{c}2.9 \% \\
(3)\end{array}$ & $\begin{array}{c}0.0 \% \\
(0)\end{array}$ & 5.25 & 105 \\
\hline $\begin{array}{l}\text { Change of } \\
\text { Management }\end{array}$ & $\begin{array}{c}1.0 \% \\
(1)\end{array}$ & \begin{tabular}{c|}
$3.8 \%$ \\
$(4)$
\end{tabular} & $\begin{array}{c}1.0 \% \\
(1)\end{array}$ & $\begin{array}{c}3.8 \% \\
(4)\end{array}$ & \begin{tabular}{|c|}
$3.8 \%$ \\
$(4)$
\end{tabular} & $\begin{array}{c}9.5 \% \\
(10)\end{array}$ & $\begin{array}{c}76.2 \% \\
(80)\end{array}$ & $\begin{array}{c}0.0 \% \\
(0)\end{array}$ & $\begin{array}{c}1.0 \% \\
(1)\end{array}$ & $\begin{array}{c}0.0 \% \\
(0)\end{array}$ & 6.45 & 105 \\
\hline $\begin{array}{l}\text { Unrealistic } \\
\text { Schedule }\end{array}$ & $\begin{array}{c}3.8 \% \\
(4)\end{array}$ & $\begin{array}{c}4.8 \% \\
(5)\end{array}$ & \begin{tabular}{|c|}
$57.1 \%$ \\
$(60)$
\end{tabular} & \begin{tabular}{|c|}
$25.7 \%$ \\
$(27)$
\end{tabular} & \begin{tabular}{|c|}
$2.9 \%$ \\
$(3)$
\end{tabular} & \begin{tabular}{|c|}
$2.9 \%$ \\
$(3)$
\end{tabular} & $\begin{array}{c}1.9 \% \\
(2) \\
\end{array}$ & $\begin{array}{c}1.0 \% \\
(1)\end{array}$ & $\begin{array}{c}0.0 \% \\
(0)\end{array}$ & $\begin{array}{c}0.0 \% \\
(0)\end{array}$ & 3.40 & 105 \\
\hline $\begin{array}{l}\text { Political } \\
\text { uncertainty }\end{array}$ & $\begin{array}{c}1.0 \% \\
(1)\end{array}$ & \begin{tabular}{c||}
$1.9 \%$ \\
$(2)$
\end{tabular} & $\begin{array}{c}1.0 \% \\
(1)\end{array}$ & $\begin{array}{c}4.8 \% \\
(5)\end{array}$ & $\begin{array}{c}17.1 \% \\
(18)\end{array}$ & \begin{tabular}{|c|}
$59.0 \%$ \\
$(62)$
\end{tabular} & $\begin{array}{c}11.4 \% \\
(12)\end{array}$ & $\begin{array}{c}1.0 \% \\
(1)\end{array}$ & $\begin{array}{c}1.0 \% \\
(1)\end{array}$ & $\begin{array}{c}1.9 \% \\
(2)\end{array}$ & 5.82 & 105 \\
\hline $\begin{array}{l}\text { Delay in } \\
\text { progress } \\
\text { payments }\end{array}$ & $\begin{array}{c}1.0 \% \\
(1)\end{array}$ & $\begin{array}{c}81.0 \% \\
(85)\end{array}$ & $\begin{array}{c}4.8 \% \\
(5)\end{array}$ & $\begin{array}{c}0.0 \% \\
(0)\end{array}$ & $\begin{array}{c}1.0 \% \\
(1)\end{array}$ & $\begin{array}{c}2.9 \% \\
(3)\end{array}$ & $\begin{array}{c}5.7 \% \\
(6)\end{array}$ & $\begin{array}{c}1.9 \% \\
(2)\end{array}$ & $\begin{array}{l}1.9 \% \\
(2)\end{array}$ & $\begin{array}{c}0.0 \% \\
(0)\end{array}$ & 2.71 & 105 \\
\hline
\end{tabular}


Causes of Schedule Overruns in Pakistani Construction Industry

\begin{tabular}{|l||c|c|c||c||c||c||c|c|c|c|c|c|}
\hline $\begin{array}{l}\text { Absence of } \\
\text { Escalation }\end{array}$ & $\begin{array}{c}0.0 \% \\
(0)\end{array}$ & $\begin{array}{c}1.9 \% \\
(2)\end{array}$ & $\begin{array}{c}1.0 \% \\
(1)\end{array}$ & $\begin{array}{c}1.0 \% \\
(1)\end{array}$ & $\begin{array}{c}0.0 \% \\
(0)\end{array}$ & $\begin{array}{c}3.8 \% \\
(4)\end{array}$ & $\begin{array}{c}1.0 \% \\
(1)\end{array}$ & $\begin{array}{c}16.2 \% \\
(17)\end{array}$ & $\begin{array}{c}73.3 \% \\
(77)\end{array}$ & $\begin{array}{c}1.9 \% \\
(2)\end{array}$ & 8.49 & 105 \\
Clause & & & & & & & & & \\
\hline High Tax on & $\begin{array}{c}0.0 \% \\
(0)\end{array}$ & $\begin{array}{c}0.0 \% \\
(0)\end{array}$ & $\begin{array}{c}1.0 \% \\
(1)\end{array}$ & $\begin{array}{c}2.9 \% \\
(3)\end{array}$ & $\begin{array}{c}0.0 \% \\
(0)\end{array}$ & $\begin{array}{c}0.0 \% \\
(0)\end{array}$ & $\begin{array}{c}1.0 \% \\
(1)\end{array}$ & $\begin{array}{c}73.3 \% \\
(77)\end{array}$ & $\begin{array}{c}18.1 \% \\
(19)\end{array}$ & $\begin{array}{c}3.8 \% \\
(4)\end{array}$ & 8.09 & 105 \\
Construction & $(0)$ & $\begin{array}{c}0.0 \% \\
\text { Political }\end{array}$ & $\begin{array}{c}1.9 \% \\
(2)\end{array}$ & $\begin{array}{c}0.0 \% \\
(0)\end{array}$ & $\begin{array}{c}1.9 \% \\
(3)\end{array}$ & $\begin{array}{c}0.0 \% \\
(0)\end{array}$ & $\begin{array}{c}0.0 \% \\
(0)\end{array}$ & $\begin{array}{c}1.0 \% \\
(1)\end{array}$ & $\begin{array}{c}1.0 \% \\
(1)\end{array}$ & $\begin{array}{c}91.4 \% \\
(96)\end{array}$ & 9.50 & 105 \\
\hline Interests & $(2)$ & $(0)$ & & \\
\hline
\end{tabular}

Table-1 Ratings of Causes

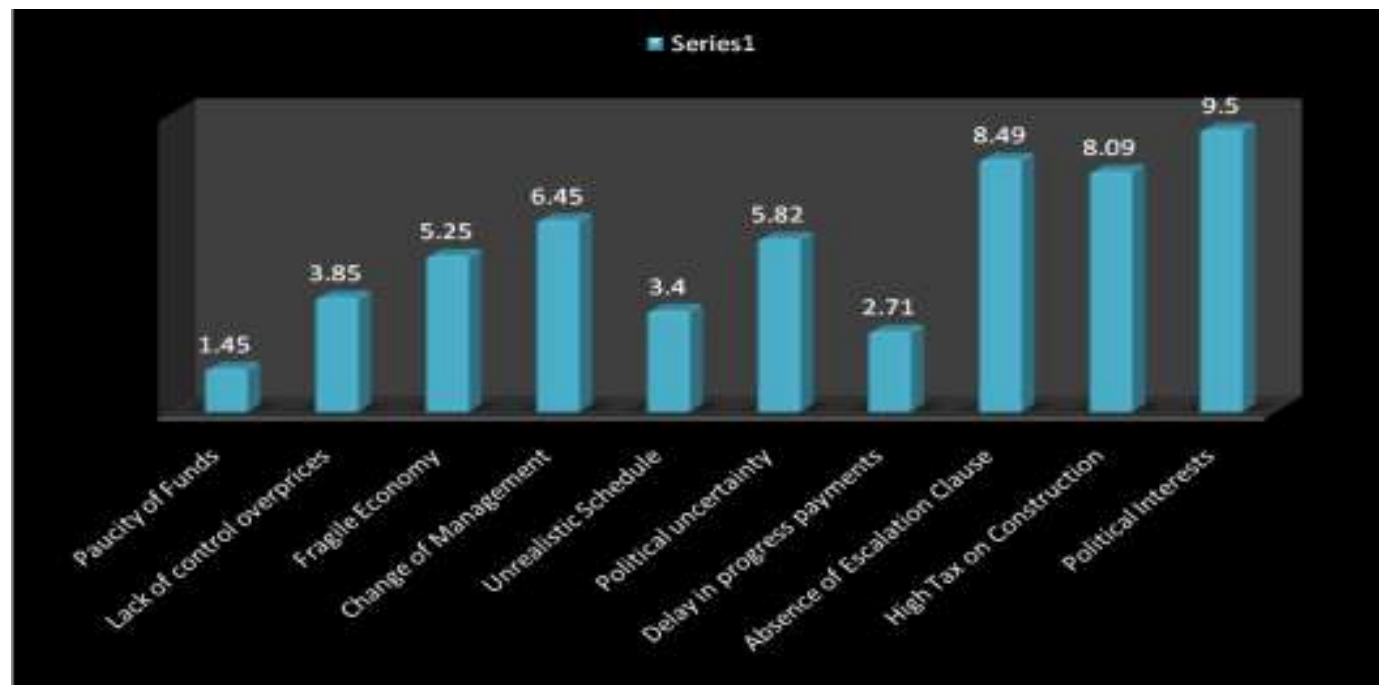

Fig 1 (a): Rankings of causes

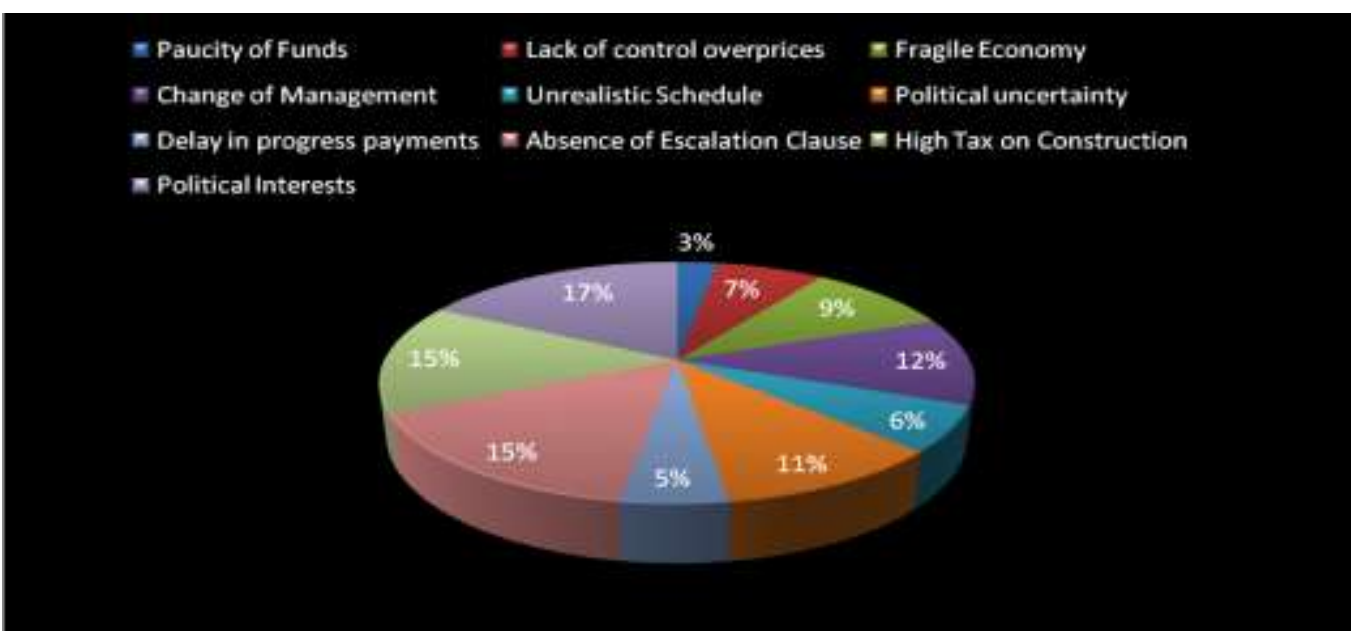

Fig 1 (b): Rankings on pie chart

5.2 Schedule Slippage due to Contractors.

This is the prime stakeholders of any construction projects, who is the actual doer or executioner entity. He implements the vision of owner on ground. Instances have been seen and data in various publications reveals that most of the contractors, do not get mobilized immediately after award of the contracts. This is definitely an unnecessary and unwanted delay which shows a callous attitude towards performance of contract and the project. The other connected to this issue is site management after getting mobilized; which includes establishment of access, routes, offices, material laboratories, Project Management Information System (PMIS), warehouse or storage, labour accommodation and supply chain for men, machines and materials. Those contractors who are weak in planning, exhibit such type of response at job sites. The issue gets aggravated when, the contractors do not possess good financial health, and capacity to manage complete range of construction activities on the projects. So eventually the quality of the project gets compromised, which culminates into disputes with suppliers and subcontractors.

It has been observed that lack of PMIS and modern construction technology like batch plants, tower cranes, cargo lifts, concrete pumps and transit mixers etcetera creates miss-coordination among internal and external 
stakeholders.

All of the above causes were made part of this survey and were annexed to this domain. Ten most occurring reasons were identified for soliciting the ranking and severity from respondents. After a thorough analysis of the data, the 103 response count of respondents rated Poor Site Management and Inefficient Planning as the top two significant causes with a response rate of 1.49 and 2.27 respectively. These results have been tabulated in Table 2 and plotted on fig $-2(\mathrm{a} \& \mathrm{~b})$ respectively.

\begin{tabular}{|c|c|c|c|c|c|c|c|c|c|c|c|c|}
\hline & 1 & 2 & 3 & 4 & 5 & 6 & 7 & 8 & 9 & 10 & $\begin{array}{c}\text { Rating } \\
\text { Average }\end{array}$ & $\begin{array}{c}\text { Response } \\
\text { Count }\end{array}$ \\
\hline $\begin{array}{l}\text { Poor Site } \\
\text { Management }\end{array}$ & $\begin{array}{c}86.4 \% \\
(89)\end{array}$ & \begin{tabular}{|c|}
$4.9 \%$ \\
$(5)$
\end{tabular} & \begin{tabular}{|c|}
$1.0 \%$ \\
$(1)$
\end{tabular} & \begin{tabular}{|c|}
$1.0 \%$ \\
$(1)$
\end{tabular} & \begin{tabular}{|c|}
$2.9 \%$ \\
$(3)$
\end{tabular} & \begin{tabular}{|c|}
$0.0 \%$ \\
$(0)$
\end{tabular} & \begin{tabular}{|c|}
$1.9 \%$ \\
$(2)$
\end{tabular} & $\begin{array}{c}1.0 \% \\
(1)\end{array}$ & $\begin{array}{c}0.0 \% \\
(0)\end{array}$ & $\begin{array}{c}1.0 \% \\
(1)\end{array}$ & 1.49 & 103 \\
\hline $\begin{array}{l}\text { Lack of } \\
\text { Construction } \\
\text { Technology }\end{array}$ & $\begin{array}{c}3.9 \% \\
(4)\end{array}$ & $\begin{array}{c}2.9 \% \\
(3)\end{array}$ & $\begin{array}{c}2.9 \% \\
(3)\end{array}$ & $\begin{array}{c}12.6 \% \\
(13)\end{array}$ & $\begin{array}{c}23.3 \% \\
(24)\end{array}$ & $\begin{array}{c}4.9 \% \\
(5)\end{array}$ & $\begin{array}{c}44.7 \% \\
(46)\end{array}$ & $\begin{array}{c}2.9 \% \\
(3)\end{array}$ & $\begin{array}{c}1.0 \% \\
(1)\end{array}$ & $\begin{array}{l}1.0 \% \\
(1)\end{array}$ & 5.69 & 103 \\
\hline Lack of Capacity & \begin{tabular}{|c|}
$1.9 \%$ \\
$(2)$
\end{tabular} & $\begin{array}{c}3.9 \% \\
(4)\end{array}$ & \begin{tabular}{|c|}
$1.9 \%$ \\
$(2)$
\end{tabular} & \begin{tabular}{|c|}
$8.7 \%$ \\
$(9)$ \\
\end{tabular} & \begin{tabular}{|c|}
$1.0 \%$ \\
$(1)$
\end{tabular} & \begin{tabular}{|c|}
$3.9 \%$ \\
$(4)$
\end{tabular} & \begin{tabular}{|c|}
$2.9 \%$ \\
$(3)$ \\
\end{tabular} & $\begin{array}{c}73.8 \% \\
(76)\end{array}$ & $\begin{array}{c}1.0 \% \\
(1)\end{array}$ & $\begin{array}{l}1.0 \% \\
(1)\end{array}$ & 7.08 & 103 \\
\hline Poor Quality & $\begin{array}{c}1.0 \% \\
(1)\end{array}$ & $\begin{array}{c}3.9 \% \\
(4)\end{array}$ & $\begin{array}{c}7.8 \% \\
(8)\end{array}$ & $\begin{array}{c}23.3 \% \\
(24)\end{array}$ & $\begin{array}{c}17.5 \% \\
(18)\end{array}$ & $\begin{array}{c}14.6 \% \\
(15)\end{array}$ & $\begin{array}{c}26.2 \% \\
(27)\end{array}$ & $\begin{array}{c}1.9 \% \\
(2)\end{array}$ & $\begin{array}{c}1.9 \% \\
(2)\end{array}$ & $\begin{array}{l}1.9 \% \\
(2)\end{array}$ & 5.36 & 103 \\
\hline Inefficient Planning & \begin{tabular}{|c|}
$4.9 \%$ \\
$(5)$
\end{tabular} & $\begin{array}{c}77.7 \% \\
(80) \\
\end{array}$ & \begin{tabular}{|c|}
$7.8 \%$ \\
$(8)$ \\
\end{tabular} & \begin{tabular}{|c|}
$5.8 \%$ \\
$(6)$ \\
\end{tabular} & \begin{tabular}{|c|}
$2.9 \%$ \\
$(3)$ \\
\end{tabular} & \begin{tabular}{|c|}
$1.0 \%$ \\
$(1)$ \\
\end{tabular} & $\begin{array}{c}0.0 \% \\
(0) \\
\end{array}$ & $\begin{array}{c}0.0 \% \\
(0)\end{array}$ & $\begin{array}{c}0.0 \% \\
(0) \\
\end{array}$ & $\begin{array}{c}0.0 \% \\
(0)\end{array}$ & 2.27 & 103 \\
\hline $\begin{array}{l}\text { Delay in Site } \\
\text { Mobilization }\end{array}$ & \begin{tabular}{|c|}
$1.0 \%$ \\
$(1)$
\end{tabular} & $\begin{array}{c}2.9 \% \\
(3)\end{array}$ & \begin{tabular}{|c|}
$73.8 \%$ \\
$(76)$
\end{tabular} & \begin{tabular}{|c|}
$7.8 \%$ \\
$(8)$
\end{tabular} & \begin{tabular}{|c|}
$4.9 \%$ \\
$(5)$
\end{tabular} & \begin{tabular}{|c|}
$2.9 \%$ \\
$(3)$
\end{tabular} & \begin{tabular}{|c|}
$1.0 \%$ \\
$(1)$
\end{tabular} & $\begin{array}{c}1.9 \% \\
(2)\end{array}$ & $\begin{array}{c}1.0 \% \\
(1)\end{array}$ & $\begin{array}{c}2.9 \% \\
\text { (3) }\end{array}$ & 3.61 & 103 \\
\hline $\begin{array}{l}\text { Poor Financial } \\
\text { Health }\end{array}$ & \begin{tabular}{|c|}
$1.0 \%$ \\
$(1)$
\end{tabular} & $\begin{array}{c}2.9 \% \\
(3) \\
\end{array}$ & \begin{tabular}{|c|}
$1.9 \%$ \\
$(2)$ \\
\end{tabular} & \begin{tabular}{|c|}
$.9 \%$ \\
$(5)$ \\
\end{tabular} & \begin{tabular}{|c|}
$42.7 \%$ \\
$(44)$ \\
\end{tabular} & \begin{tabular}{|c|}
$40.8 \%$ \\
$(42)$ \\
\end{tabular} & \begin{tabular}{|c|}
$5.8 \%$ \\
$(6)$ \\
\end{tabular} & $\begin{array}{c}0.0 \% \\
(0) \\
\end{array}$ & $\begin{array}{c}0.0 \% \\
(0) \\
\end{array}$ & $\begin{array}{c}0.0 \% \\
(0) \\
\end{array}$ & 5.31 & 103 \\
\hline $\begin{array}{l}\text { Disputes with Sub- } \\
\text { Contractors }\end{array}$ & $\begin{array}{c}0.0 \% \\
(0) \\
\end{array}$ & $\begin{array}{c}0.0 \% \\
(0)\end{array}$ & \begin{tabular}{|c|}
$1.0 \%$ \\
$(1)$ \\
\end{tabular} & \begin{tabular}{|c|}
$0.0 \%$ \\
$(0)$ \\
\end{tabular} & \begin{tabular}{|c|}
$2.9 \%$ \\
$(3)$ \\
\end{tabular} & \begin{tabular}{|c|}
$1.0 \%$ \\
$(1)$ \\
\end{tabular} & \begin{tabular}{|c|}
$1.9 \%$ \\
$(2)$ \\
\end{tabular} & $\begin{array}{c}6.8 \% \\
(7) \\
\end{array}$ & $\begin{array}{c}84.5 \% \\
(87) \\
\end{array}$ & $\begin{array}{c}1.9 \% \\
(2)\end{array}$ & 8.71 & 103 \\
\hline Lack of PMIS & \begin{tabular}{|c|}
$0.0 \%$ \\
$(0)$
\end{tabular} & $\begin{array}{c}1.0 \% \\
(1)\end{array}$ & \begin{tabular}{|c|}
$1.0 \%$ \\
$(1)$
\end{tabular} & $\begin{array}{c}33.0 \% \\
(34)\end{array}$ & \begin{tabular}{|c|}
$1.0 \%$ \\
$(1)$ \\
\end{tabular} & \begin{tabular}{|c|}
$30.1 \%$ \\
$(31)$
\end{tabular} & \begin{tabular}{|c|}
$14.6 \%$ \\
$(15)$
\end{tabular} & $\begin{array}{c}10.7 \% \\
(11)\end{array}$ & $\begin{array}{c}7.8 \% \\
(8)\end{array}$ & $\begin{array}{l}1.0 \% \\
(1)\end{array}$ & 5.89 & 103 \\
\hline $\begin{array}{l}\text { Poor } \\
\text { Communications }\end{array}$ & \begin{tabular}{|c|}
$0.0 \%$ \\
$(0)$
\end{tabular} & $\begin{array}{c}0.0 \% \\
(0)\end{array}$ & \begin{tabular}{|c|}
$1.0 \%$ \\
$(1)$
\end{tabular} & \begin{tabular}{|c|}
$2.9 \%$ \\
$(3)$ \\
\end{tabular} & \begin{tabular}{|c|}
$1.0 \%$ \\
$(1)$ \\
\end{tabular} & \begin{tabular}{|c|}
$1.0 \%$ \\
$(1)$
\end{tabular} & $\begin{array}{c}1.0 \% \\
(1)\end{array}$ & $\begin{array}{c}1.0 \% \\
(1)\end{array}$ & $\begin{array}{c}2.9 \% \\
(3)\end{array}$ & $\begin{array}{l}89.3 \% \\
(92)\end{array}$ & 9.59 & 103 \\
\hline
\end{tabular}

Table-2 Ratings of Causes

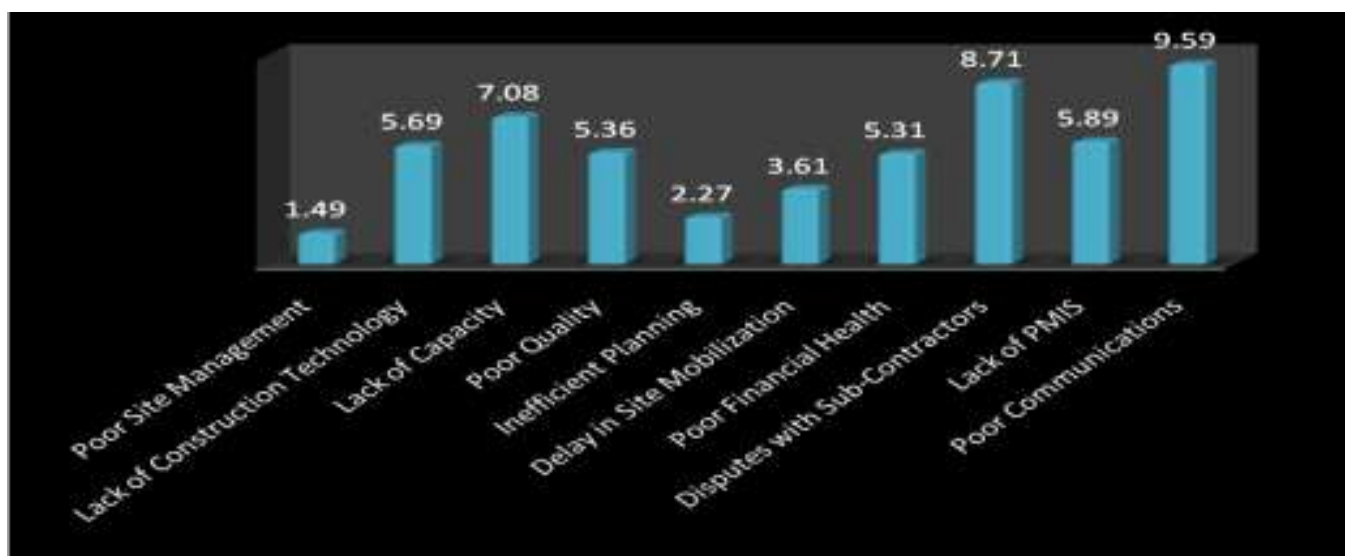

Fig 2 (a): Rankings on causes

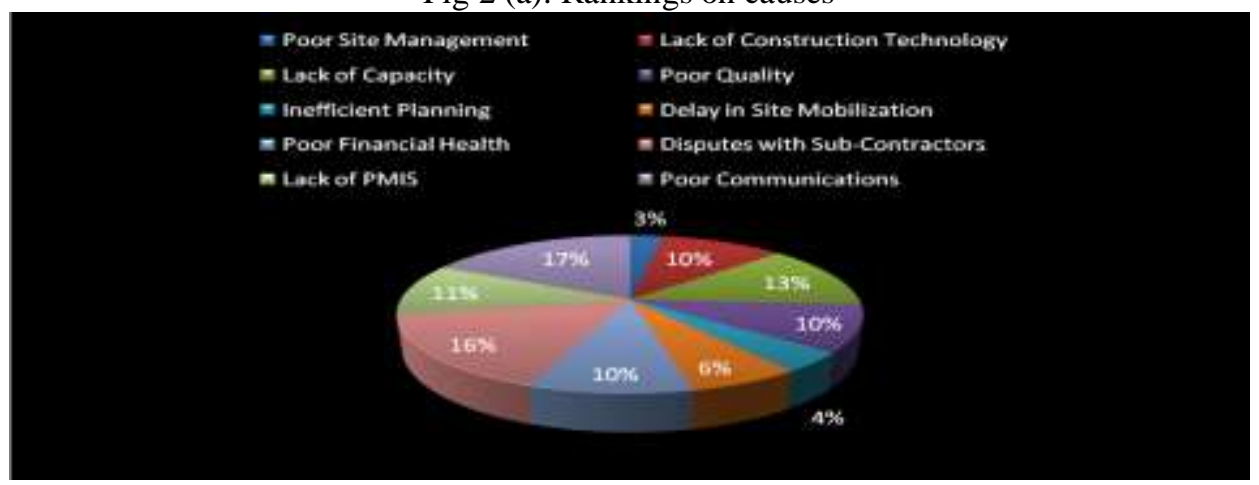

Fig 2 (b): Rankings on pie chart 
5.3 Schedule Slippage due to Consultants. In the construction industry of Pakistan, there is a general perception that consultants (both the design professionals and the architects) enjoy ultimate verdict and decision power for the constructability issues on the projects. It is unwise to say that contractors are the sole entities who are to be blamed for delay, failure or abandonment of construction projects.

If objectively analyzed, consultants have direct influence and effect over success or failure of construction projects. Their professional or unprofessional actions do lay good or bad repercussions onto the contractors and the projects alike. For instance, when their will be clash and conflict among structural, public health works, electric and water supply drawings with architectural drawings, the ultimate problem will be shifted to the contractor. He may not be able to execute the drawings due to design errors, missing or insufficient data, and lack of integration of subsidiary plans.

This situation is further exploited when the consultants either do not give approval of shop drawings prepared by the contractors or altogether reject them.

Similarly there is a common trend of hiring few Auto CAD operators for preparing drawings and their supporting sections and elevations. They are neither engineers or architects, nor they are authorized to prepare drawings on their own. They are in fact, helpers for the design professionals and the architects. Hence total reliance on CAD operators will lead to constructability issues on the jobsites.

Being professionals, it becomes imperative to implement an open door policy, so that every stakeholder can access for seeking guidance and consultation pertaining to the projects. Unfortunately there is a huge communication gap among primary stakeholders. That is why, certain consultants add extra work at their will, just to pad up engineer estimates, without the approval of the clients or owners. This practice culminates into conflicts among contractors, sub contractors, clients and the consultants themselves.

The causes as highlighted above, were grouped into this bracket of the questionnaire and accordingly respondents were requested for furnishing their expert opinion on each cause. Overall, the response count in this bracket was 103. The results indicate that Design Errors and Delay in Approvals are the top two significant causes with an average ratings of 1.57 and 2.12 respectively. Results of all ten causes of this category have been compiled in following tables and graphs.

\begin{tabular}{|c|c|c|c|c|c|c|c|c|c|c|c|c|}
\hline & 1 & 2 & 3 & 4 & 5 & 6 & 7 & 8 & 9 & 10 & $\begin{array}{l}\text { Rating } \\
\text { Average }\end{array}$ & $\begin{array}{c}\text { Response } \\
\text { Count }\end{array}$ \\
\hline Unqualified Staff & $\begin{array}{c}3.9 \% \\
(4)\end{array}$ & \begin{tabular}{|c|}
$2.9 \%$ \\
$(3)$ \\
\end{tabular} & \begin{tabular}{|c|}
$2.9 \%$ \\
$(3)$ \\
\end{tabular} & $\begin{array}{c}2.9 \% \\
(3)\end{array}$ & $\begin{array}{c}0.0 \% \\
(0)\end{array}$ & $\begin{array}{c}2.9 \% \\
(3)\end{array}$ & $\begin{array}{c}33.0 \% \\
(34)\end{array}$ & $\begin{array}{c}35.0 \% \\
(36)\end{array}$ & $\begin{array}{c}16.5 \% \\
(17)\end{array}$ & $\begin{array}{c}0.0 \% \\
(0)\end{array}$ & 7.07 & 103 \\
\hline $\begin{array}{l}\text { Delay in } \\
\text { Approvals }\end{array}$ & $\begin{array}{c}6.8 \% \\
(7)\end{array}$ & $\begin{array}{c}86.4 \% \\
(89) \\
\end{array}$ & $\begin{array}{c}1.9 \% \\
(2)\end{array}$ & $\begin{array}{c}1.0 \% \\
(1)\end{array}$ & $\begin{array}{l}1.9 \% \\
(2)\end{array}$ & $\begin{array}{c}1.0 \% \\
(1)\end{array}$ & $\begin{array}{c}1.0 \% \\
(1)\end{array}$ & $\begin{array}{c}0.0 \% \\
(0)\end{array}$ & $\begin{array}{c}0.0 \% \\
(0)\end{array}$ & $\begin{array}{c}0.0 \% \\
(0)\end{array}$ & 2.12 & 103 \\
\hline Rigid Attitude & $\begin{array}{c}1.9 \% \\
(2)\end{array}$ & $\begin{array}{c}1.9 \% \\
(2)\end{array}$ & $\begin{array}{c}1.9 \% \\
(2) \\
\end{array}$ & $\begin{array}{c}1.9 \% \\
(2)\end{array}$ & $\begin{array}{l}2.9 \% \\
(3)\end{array}$ & $\begin{array}{c}1.0 \% \\
(1)\end{array}$ & $\begin{array}{c}1.9 \% \\
(2) \\
\end{array}$ & $\begin{array}{c}2.9 \% \\
(3) \\
\end{array}$ & $\begin{array}{c}1.0 \% \\
(1)\end{array}$ & $\begin{array}{c}82.5 \% \\
(85)\end{array}$ & 9.11 & 103 \\
\hline$\overline{\text { Desig }}$ & $\begin{array}{c}82.5 \% \\
(85)\end{array}$ & \begin{tabular}{|c|}
$1.9 \%$ \\
$(2)$
\end{tabular} & \begin{tabular}{|c|}
$3.9 \%$ \\
$(4)$
\end{tabular} & $\begin{array}{c}4.9 \% \\
(5)\end{array}$ & $\begin{array}{c}2.9 \% \\
(3)\end{array}$ & $\begin{array}{c}2.9 \% \\
(3)\end{array}$ & \begin{tabular}{|c|}
$0.0 \%$ \\
$(0)$
\end{tabular} & $\begin{array}{c}1.0 \% \\
(1)\end{array}$ & $\begin{array}{c}0.0 \% \\
(0)\end{array}$ & $\begin{array}{c}0.0 \% \\
(0)\end{array}$ & 1.57 & 103 \\
\hline $\begin{array}{l}\text { Lack of Modern } \\
\text { Design } \\
\text { Technology } \\
\end{array}$ & $\begin{array}{c}0.0 \% \\
(0)\end{array}$ & \begin{tabular}{|c|}
$1.9 \%$ \\
$(2)$
\end{tabular} & $\begin{array}{c}1.9 \% \\
(2)\end{array}$ & $\begin{array}{c}3.9 \% \\
(4)\end{array}$ & $\begin{array}{l}5.8 \% \\
(6)\end{array}$ & $\begin{array}{c}1.0 \% \\
(1)\end{array}$ & \begin{tabular}{|c|}
$1.9 \%$ \\
$(2)$
\end{tabular} & $\begin{array}{c}18.4 \% \\
(19)\end{array}$ & $\begin{array}{c}63.1 \% \\
(65)\end{array}$ & $\begin{array}{l}1.9 \% \\
(2)\end{array}$ & 8.09 & 103 \\
\hline $\begin{array}{l}\text { Poor } \\
\text { Communications }\end{array}$ & $\begin{array}{c}0.0 \% \\
(0)\end{array}$ & $\begin{array}{c}1.9 \% \\
(2)\end{array}$ & \begin{tabular}{|c|}
$7.8 \%$ \\
$(8)$ \\
\end{tabular} & $\begin{array}{c}47.6 \% \\
(49)\end{array}$ & $\begin{array}{c}30.1 \% \\
(31)\end{array}$ & $\begin{array}{c}4.9 \% \\
(5)\end{array}$ & $\begin{array}{c}1.9 \% \\
(2) \\
\end{array}$ & $\begin{array}{c}3.9 \% \\
(4)\end{array}$ & $\begin{array}{c}0.0 \% \\
(0)\end{array}$ & $\begin{array}{c}1.9 \% \\
(2)\end{array}$ & 4.61 & 103 \\
\hline $\begin{array}{l}\text { Extra Work } \\
\text { without Approval }\end{array}$ & $\begin{array}{c}0.0 \% \\
(0)\end{array}$ & $\begin{array}{c}1.0 \% \\
(1)\end{array}$ & $\begin{array}{c}1.9 \% \\
(2)\end{array}$ & $\begin{array}{l}34.0 \% \\
(35)\end{array}$ & $\begin{array}{l}46.6 \% \\
(48)\end{array}$ & $\begin{array}{c}4.9 \% \\
(5)\end{array}$ & \begin{tabular}{|c|}
$2.9 \%$ \\
$(3)$
\end{tabular} & \begin{tabular}{|c|}
$2.9 \%$ \\
$(3)$
\end{tabular} & $\begin{array}{c}2.9 \% \\
(3)\end{array}$ & $\begin{array}{c}2.9 \% \\
(3)\end{array}$ & 5.05 & 103 \\
\hline Insufficient Data & $\begin{array}{c}1.9 \% \\
(2)\end{array}$ & $\begin{array}{c}0.0 \% \\
(0)\end{array}$ & $\begin{array}{c}2.9 \% \\
(3)\end{array}$ & $\begin{array}{c}2.9 \% \\
(3)\end{array}$ & $\begin{array}{l}6.8 \% \\
(7)\end{array}$ & $\begin{array}{l}71.8 \% \\
(74)\end{array}$ & $\begin{array}{c}5.8 \% \\
(6) \\
\end{array}$ & $\begin{array}{c}1.9 \% \\
(2)\end{array}$ & $\begin{array}{c}4.9 \% \\
(5)\end{array}$ & $\begin{array}{c}1.0 \% \\
(1)\end{array}$ & 5.97 & 103 \\
\hline $\begin{array}{l}\text { Conflicts with } \\
\text { Contractors }\end{array}$ & $\begin{array}{c}1.0 \% \\
(1)\end{array}$ & $\begin{array}{c}0.0 \% \\
(0)\end{array}$ & $\begin{array}{c}1.0 \% \\
(1)\end{array}$ & $\begin{array}{c}1.0 \% \\
(1)\end{array}$ & $\begin{array}{c}0.0 \% \\
(0)\end{array}$ & $\begin{array}{c}7.8 \% \\
(8)\end{array}$ & $\begin{array}{c}48.5 \% \\
(50)\end{array}$ & $\begin{array}{c}34.0 \% \\
(35)\end{array}$ & $\begin{array}{c}3.9 \% \\
(4)\end{array}$ & $\begin{array}{c}2.9 \% \\
(3)\end{array}$ & 7.30 & 103 \\
\hline $\begin{array}{l}\text { Lack of Project } \\
\text { Integration }\end{array}$ & $\begin{array}{c}1.9 \% \\
(2)\end{array}$ & \begin{tabular}{|c|}
$1.9 \%$ \\
$(2)$
\end{tabular} & $\begin{array}{c}73.8 \% \\
(76)\end{array}$ & $\begin{array}{c}0.0 \% \\
(0)\end{array}$ & $\begin{array}{l}2.9 \% \\
(3)\end{array}$ & $\begin{array}{c}1.9 \% \\
(2)\end{array}$ & \begin{tabular}{|c|}
$2.9 \%$ \\
$(3)$ \\
\end{tabular} & $\begin{array}{c}0.0 \% \\
(0)\end{array}$ & $\begin{array}{c}7.8 \% \\
(8)\end{array}$ & $\begin{array}{c}6.8 \% \\
(7)\end{array}$ & 4.12 & 103 \\
\hline
\end{tabular}

Table-3 Ratings of Causes 


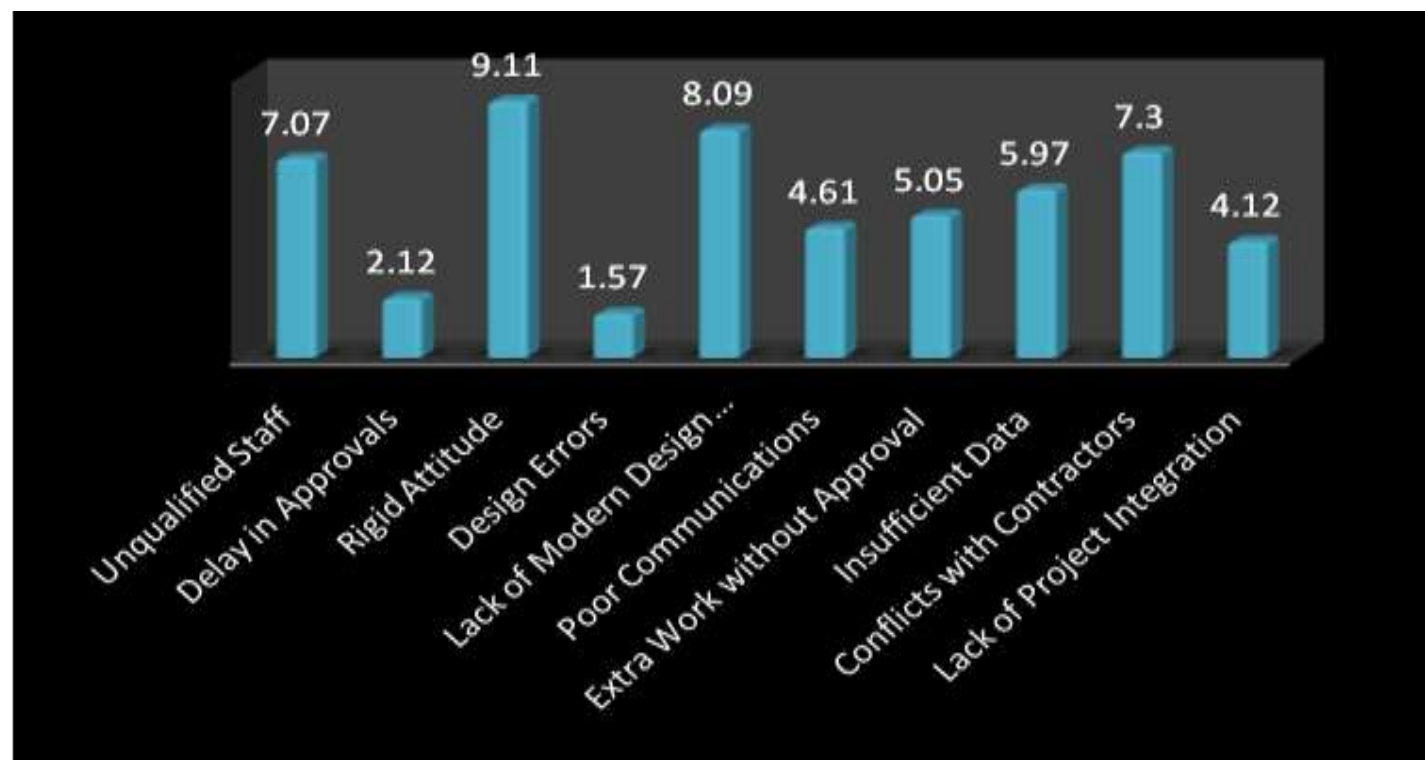

Fig 3 (a): Rankings of causes

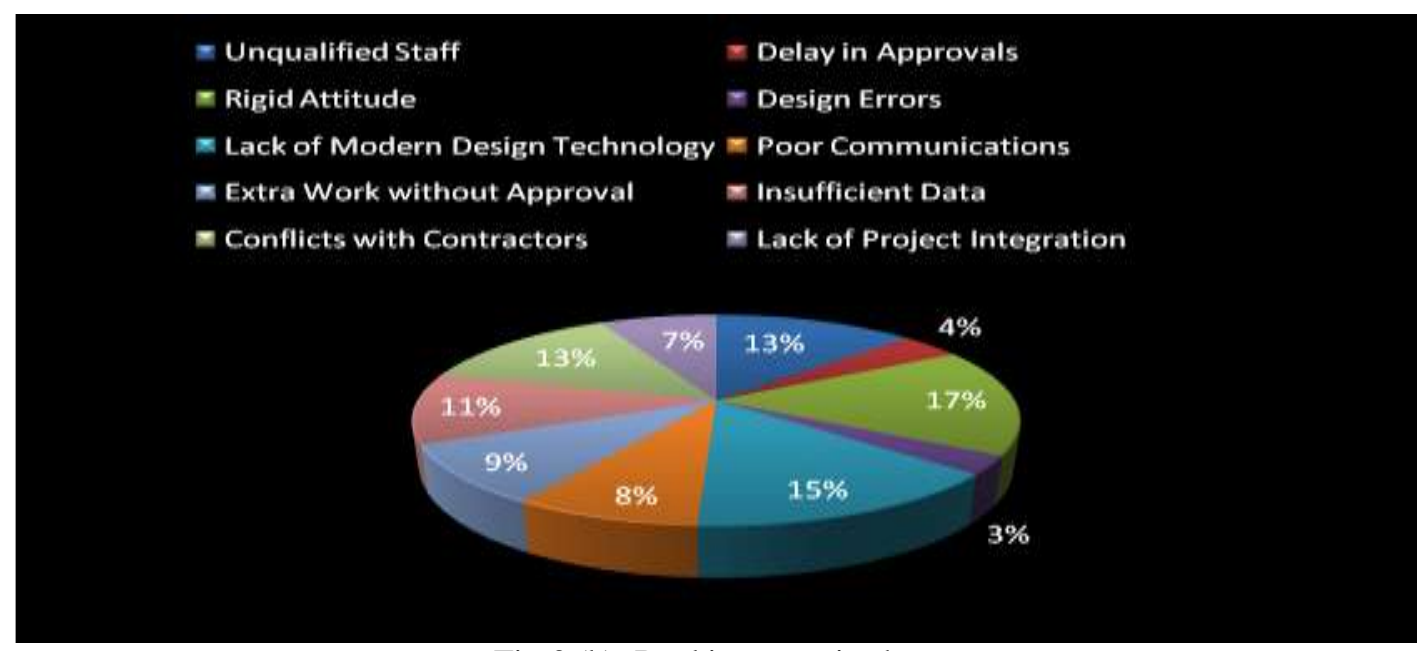

Fig 3 (b): Rankings on pie chart

5.4 Schedule Slippage due to Clients / Owners.

This is the main and very important project stakeholder, who conceives the project, approves the project charter and subsequently accords approvals and disburses the progress payment to the contractors.

The involvement of the client in all phases of the project i.e. entire project life cycle is mandatory, in order to make the project a complete success. Unfortunately there are member of causes and reasons, over which the clients do not play their due role and input into the development and growth of the project. In the succeeding paragraphs, the authors have described the rundown of such factors.

Timely funding on the project is very important in order to expedite the progress as per approved construction schedule and schedule baseline. Failure to ensure a smooth cash flow without any plausible reasons will halt and disrupt the project momentum. Most of the projects fail due to disruption in cash flows.

The project curve takes off very rapidly during execution phase and efforts should be made to maintain, this uplift trend. Unfortunately majority of the clients, ask for major changes during this phase. This speaks of their level of involvement during planning stage. Planning is such a phase where there is a lot of room for major modifications, variations, deviations or additions in the scope but not in execution phase. These changes entail heavy expenditures and claims by contractor which over budget the cost baselines.

Similarly, clients also demand extra works, specifications and features in the project design without incurring additional costs. This behaviour is known as "Gold Plating" in the language of Project Management. When this occurs, the contractor compromises the quality in other field just to please the client.

Like consultants, clients also do not adhere to the approved timelines and do not approve the change orders timely. A slow decision in one activity pushes the next activity and subsequently it becomes a chain reaction for the project as a whole. 
It is very important for the benefit of the project, that there must be adequate funds for maintenance of cash flows as well as approvals of necessary change orders. Moreover during planning, all requirements should be well briefed without leaving any ambiguity, for later phases.

Establishment of proper communication management and mechanism eradicates most of the problems and issues related to the project. This aspect then finally helps in smooth user acceptance at the completion of the project.

Under this segment, a total of ten causes were tabulated as described above for soliciting input from respondents. The response count in this domain was 101. The most significant cause which received highest rating (2.74) was Disruption in Cash flows. The next causes with a rating of 3.46 each were, Lack of Involvement in Planning Phase and Insufficient Funds. It is true that without detailed planning and uninterrupted cash flow, no project can be a complete success. Neither can it be completed on time nor on the approved budget.

The data on remaining causes is appended in table 4 which has been represented in the graphical form as described in figure $4(\mathrm{a} \& \mathrm{~b})$.

\begin{tabular}{|c|c|c|c|c|c|c|c|c|c|c|c|c|}
\hline & 1 & 2 & 3 & 4 & 5 & 6 & 7 & 8 & 9 & 10 & $\begin{array}{l}\text { Rating } \\
\text { Average }\end{array}$ & \begin{tabular}{|c|} 
Response \\
Count \\
\end{tabular} \\
\hline \begin{tabular}{|l|} 
Frequent \\
Changes during \\
Execution
\end{tabular} & $\begin{array}{l}9.9 \% \\
(10)\end{array}$ & $\begin{array}{l}9.9 \% \\
(10)\end{array}$ & $\begin{array}{c}6.9 \% \\
(7)\end{array}$ & $\begin{array}{l}55.4 \% \\
(56)\end{array}$ & $\begin{array}{c}8.9 \% \\
(9)\end{array}$ & $\begin{array}{l}2.0 \% \\
(2)\end{array}$ & $\begin{array}{c}2.0 \% \\
(2)\end{array}$ & $\begin{array}{c}3.0 \% \\
(3)\end{array}$ & $\begin{array}{c}1.0 \% \\
(1)\end{array}$ & $\begin{array}{c}1.0 \% \\
(1)\end{array}$ & 3.85 & 101 \\
\hline $\begin{array}{l}\text { Lack of } \\
\text { Involvement in } \\
\text { Planning stage }\end{array}$ & $\begin{array}{l}8.9 \% \\
(9)\end{array}$ & $\begin{array}{l}5.9 \% \\
(6)\end{array}$ & $\begin{array}{l}61.4 \% \\
(62)\end{array}$ & $\begin{array}{c}7.9 \% \\
(8)\end{array}$ & $\begin{array}{c}5.0 \% \\
(5)\end{array}$ & $\begin{array}{c}2.0 \% \\
(2)\end{array}$ & $\begin{array}{c}4.0 \% \\
(4)\end{array}$ & $\begin{array}{c}1.0 \% \\
(1)\end{array}$ & $\begin{array}{c}3.0 \% \\
(3)\end{array}$ & $\begin{array}{c}1.0 \% \\
(1)\end{array}$ & 3.46 & 101 \\
\hline $\begin{array}{l}\text { Gold } 1 \\
\text { from }\end{array}$ & $\begin{array}{c}4.0 \% \\
(4)\end{array}$ & $\begin{array}{c}4.0 \% \\
(4)\end{array}$ & $\begin{array}{c}5.0 \% \\
(5)\end{array}$ & $\begin{array}{c}2.0 \% \\
(2)\end{array}$ & $\begin{array}{c}4.0 \% \\
(4)\end{array}$ & $\begin{array}{c}1.0 \% \\
(1)\end{array}$ & $\begin{array}{c}8.9 \% \\
(9)\end{array}$ & $\begin{array}{c}7.9 \% \\
(8)\end{array}$ & $\begin{array}{c}60.4 \% \\
(61)\end{array}$ & $\begin{array}{c}3.0 \% \\
(3)\end{array}$ & .59 & 01 \\
\hline $\begin{array}{l}\text { Slowness in } \\
\text { Decision } \\
\text { Making } \\
\end{array}$ & $\begin{array}{l}4.0 \% \\
(4)\end{array}$ & $\begin{array}{c}6.9 \% \\
(7)\end{array}$ & $\begin{array}{c}10.9 \% \\
(11)\end{array}$ & $\begin{array}{l}9.9 \% \\
(10)\end{array}$ & $\begin{array}{l}53.5 \% \\
(54)\end{array}$ & $\begin{array}{c}10.9 \% \\
(11)\end{array}$ & $\begin{array}{c}2.0 \% \\
(2)\end{array}$ & $\begin{array}{c}0.0 \% \\
(0)\end{array}$ & $\begin{array}{c}2.0 \% \\
(2)\end{array}$ & $\begin{array}{c}0.0 \% \\
(0)\end{array}$ & 4.54 & $\overline{01}$ \\
\hline $\begin{array}{l}\text { Change of } \\
\text { Design }\end{array}$ & $\begin{array}{c}3.0 \% \\
(3)\end{array}$ & $\begin{array}{c}4.0 \% \\
(4)\end{array}$ & $\begin{array}{c}5.0 \% \\
(5)\end{array}$ & $\begin{array}{c}5.9 \% \\
(6)\end{array}$ & $\begin{array}{l}12.9 \% \\
(13)\end{array}$ & $\begin{array}{c}18.8 \% \\
(19)\end{array}$ & $\begin{array}{l}48.5 \% \\
(49)\end{array}$ & $\begin{array}{c}2.0 \% \\
(2)\end{array}$ & $\begin{array}{c}0.0 \% \\
(0)\end{array}$ & $\begin{array}{c}0.0 \% \\
(0)\end{array}$ & 5.82 & 101 \\
\hline $\begin{array}{l}\text { Disruption in } \\
\text { Cash-flows }\end{array}$ & $\begin{array}{c}63.4 \% \\
(64)\end{array}$ & $\begin{array}{c}3.0 \% \\
(3)\end{array}$ & $\begin{array}{c}1.0 \% \\
(1)\end{array}$ & $\begin{array}{c}6.9 \% \\
(7)\end{array}$ & $\begin{array}{c}5.9 \% \\
(6)\end{array}$ & $\begin{array}{c}7.9 \% \\
(8)\end{array}$ & $\begin{array}{c}3.0 \% \\
(3)\end{array}$ & $\begin{array}{c}4.0 \% \\
(4)\end{array}$ & $\begin{array}{c}5.0 \% \\
(5)\end{array}$ & $\begin{array}{c}0.0 \% \\
(0)\end{array}$ & 2.74 & 01 \\
\hline $\begin{array}{l}\text { Poor } \\
\text { Communications }\end{array}$ & $\begin{array}{c}0.0 \% \\
(0)\end{array}$ & $\begin{array}{c}4.0 \% \\
(4)\end{array}$ & $\begin{array}{c}1.0 \% \\
(1)\end{array}$ & $\begin{array}{c}4.0 \% \\
(4)\end{array}$ & $\begin{array}{c}2.0 \% \\
(2)\end{array}$ & $\begin{array}{c}10.9 \% \\
(11)\end{array}$ & $\begin{array}{c}13.9 \% \\
(14)\end{array}$ & $\begin{array}{c}62.4 \% \\
(63)\end{array}$ & $\begin{array}{c}1.0 \% \\
(1)\end{array}$ & $\begin{array}{c}1.0 \% \\
(1)\end{array}$ & 7.17 & 10 \\
\hline $\begin{array}{l}\text { Insufficient } \\
\text { Funds }\end{array}$ & $\begin{array}{c}6.9 \% \\
(7) \\
\end{array}$ & $\begin{array}{c}57.4 \% \\
(58) \\
\end{array}$ & $\begin{array}{c}5.0 \% \\
(5) \\
\end{array}$ & $\begin{array}{c}3.0 \% \\
(3) \\
\end{array}$ & $\begin{array}{c}5.9 \% \\
(6) \\
\end{array}$ & $\begin{array}{c}5.0 \% \\
(5) \\
\end{array}$ & $\begin{array}{c}3.0 \% \\
(3) \\
\end{array}$ & $\begin{array}{c}8.9 \% \\
(9) \\
\end{array}$ & $\begin{array}{c}4.0 \% \\
(4) \\
\end{array}$ & $\begin{array}{c}1.0 \% \\
(1) \\
\end{array}$ & 3.46 & 101 \\
\hline $\begin{array}{l}\text { Insufficient } \\
\text { Requirements }\end{array}$ & $\begin{array}{c}0.0 \% \\
(0)\end{array}$ & $\begin{array}{c}5.0 \% \\
(5)\end{array}$ & $\begin{array}{c}3.0 \% \\
(3) \\
\end{array}$ & $\begin{array}{c}3.0 \% \\
(3) \\
\end{array}$ & $\begin{array}{c}2.0 \% \\
(2) \\
\end{array}$ & $\begin{array}{c}40.6 \% \\
(41) \\
\end{array}$ & $\begin{array}{c}14.9 \% \\
(15)\end{array}$ & $\begin{array}{c}7.9 \% \\
(8) \\
\end{array}$ & $\begin{array}{c}18.8 \% \\
(19)\end{array}$ & $\begin{array}{c}5.0 \% \\
(5) \\
\end{array}$ & 6.70 & 101 \\
\hline $\begin{array}{l}\text { Handing Taking } \\
\text { Charge }\end{array}$ & $\begin{array}{c}0.0 \% \\
(0)\end{array}$ & $\begin{array}{c}0.0 \% \\
(0)\end{array}$ & $\begin{array}{c}1.0 \% \\
(1)\end{array}$ & $\begin{array}{c}2.0 \% \\
(2)\end{array}$ & $\begin{array}{c}0.0 \% \\
(0)\end{array}$ & $\begin{array}{c}1.0 \% \\
(1)\end{array}$ & $\begin{array}{c}0.0 \% \\
(0)\end{array}$ & $\begin{array}{c}3.0 \% \\
(3)\end{array}$ & $\begin{array}{c}5.0 \% \\
(5)\end{array}$ & $\begin{array}{l}88.1 \% \\
(89)\end{array}$ & 9.66 & 101 \\
\hline
\end{tabular}

Table-4 Ratings of Causes

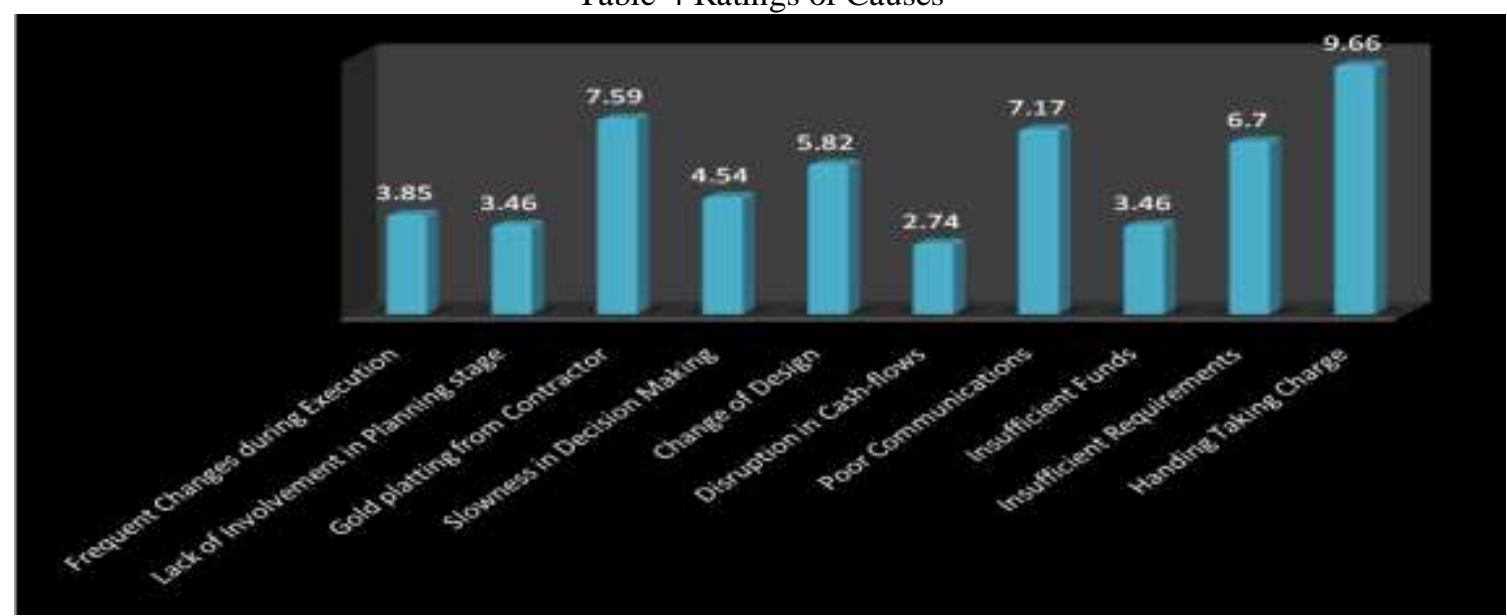

Fig 4 (a): Rankings of Causes 


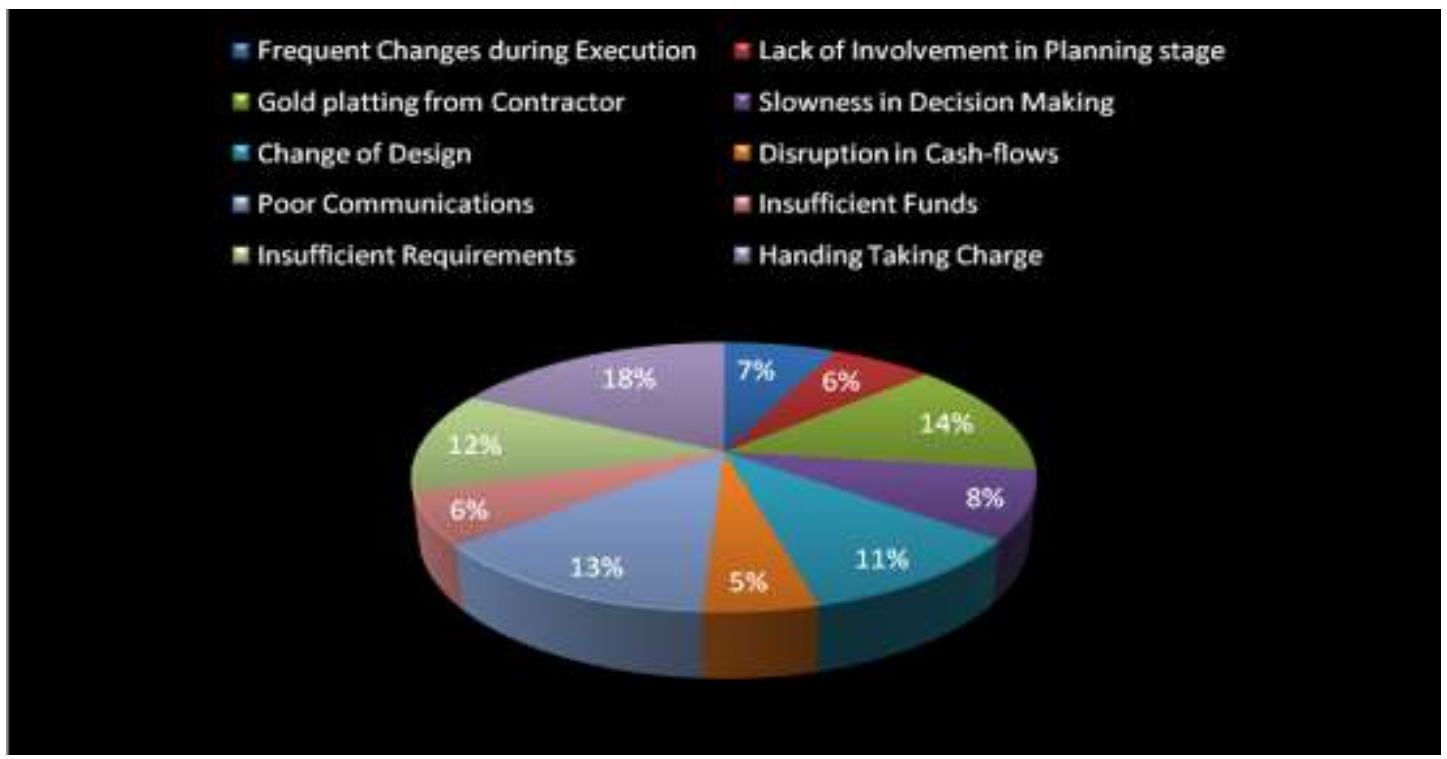

Fig 4 (b): Rankings on pie chart

5.5 Schedule Slippage due to Other Factors. Project external environment also occupies a significant space in the project schedule baseline. The schedule baseline or commonly known as completion date is affected by numerous external factors.

After thorough analysis of completed projects and the literature review, ten most occurring causes were segregated and made part of this survey under this domain.

Globalization have not only brought prosperity for the world, but it has resulted into sufferings as well. With the advent of soft borders, easy trading and elimination of unnecessary barriers in the visa policies and immigration, the world is now-a-days the facing worst menace of global terrorism. Pakistan is the frontline in the war on terror and the country has suffered enormously in economy as well as developmental projects. Today Law and Order is a big issue which has crippled the country's economy and infrastructure development.

This situation is even worsening because of natural calamities like earth quakes, floods and weather modifications. All of these factors have deep influence over construction projects.

If it is seen at micro level, there are certain issues which also contribute towards dip in the schedule baselines. There are; limited access to the site, shallow water table, and safety and health issues at job sites.

The overall response count in this domain was 99. Law and Order received highest rating of 3.22 while safety and health at the site was second highest with a rating score of 3.37. Table 5 and fig- 5 (a\&b) show the complete ratings and rankings.

\begin{tabular}{|c|c|c|c|c|c|c|c|c|c|c|c|c|}
\hline & 1 & 2 & 3 & 4 & 5 & 6 & 7 & 8 & 9 & 10 & $\begin{array}{l}\text { Rating } \\
\text { Average }\end{array}$ & $\begin{array}{c}\text { Response } \\
\text { Count }\end{array}$ \\
\hline $\begin{array}{l}\text { eme } \\
\text { ther } \\
\text { ditions }\end{array}$ & $\begin{array}{c}3.0 \% \\
(3)\end{array}$ & $\begin{array}{c}11.1 \% \\
(11)\end{array}$ & $\begin{array}{c}4.0 \% \\
(4)\end{array}$ & $\begin{array}{c}27.3 \% \\
(27)\end{array}$ & $\begin{array}{c}27.3 \% \\
(27)\end{array}$ & $\begin{array}{c}9.1 \% \\
(9)\end{array}$ & $\begin{array}{c}12.1 \% \\
(12)\end{array}$ & $\begin{array}{c}2.0 \% \\
(2)\end{array}$ & $\begin{array}{c}1.0 \% \\
(1)\end{array}$ & $\begin{array}{c}3.0 \% \\
(3)\end{array}$ & 4.78 & 99 \\
\hline Law and Order & $\begin{array}{c}18.2 \% \\
(18)\end{array}$ & $\begin{array}{c}28.3 \% \\
(28)\end{array}$ & $\begin{array}{c}6.1 \% \\
(6)\end{array}$ & $\begin{array}{c}23.2 \% \\
(23)\end{array}$ & $\begin{array}{c}18.2 \% \\
(18)\end{array}$ & $\begin{array}{c}1.0 \% \\
(1)\end{array}$ & $\begin{array}{c}2.0 \% \\
(2)\end{array}$ & $\begin{array}{c}2.0 \% \\
(2)\end{array}$ & $\begin{array}{c}1.0 \% \\
(1)\end{array}$ & $\begin{array}{c}0.0 \% \\
(0)\end{array}$ & 3.22 & 99 \\
\hline Natu & $\begin{array}{c}4.0 \% \\
(4)\end{array}$ & $\begin{array}{c}2.0 \% \\
(2)\end{array}$ & $\begin{array}{c}7.1 \% \\
(7)\end{array}$ & $\begin{array}{c}5.1 \% \\
(5)\end{array}$ & $\begin{array}{c}3.0 \% \\
(3)\end{array}$ & $\begin{array}{c}8.1 \% \\
(8)\end{array}$ & $\begin{array}{c}2.0 \% \\
(2)\end{array}$ & $\begin{array}{c}4.0 \% \\
(4)\end{array}$ & $\begin{array}{c}4.0 \% \\
(4)\end{array}$ & $\begin{array}{c}60.6 \% \\
(60)\end{array}$ & 8.02 & 99 \\
\hline $\begin{array}{l}\text { Public Non- } \\
\text { cooperation }\end{array}$ & $\begin{array}{c}3.0 \% \\
(3)\end{array}$ & $\begin{array}{c}4.0 \% \\
(4)\end{array}$ & $\begin{array}{c}7.1 \% \\
(7)\end{array}$ & $\begin{array}{c}7.1 \% \\
(7)\end{array}$ & $\begin{array}{c}3.0 \% \\
(3)\end{array}$ & $\begin{array}{c}10.1 \% \\
(10)\end{array}$ & $\begin{array}{c}9.1 \% \\
(9)\end{array}$ & $\begin{array}{c}51.5 \% \\
(51)\end{array}$ & $\begin{array}{c}4.0 \% \\
(4)\end{array}$ & $\begin{array}{c}1.0 \% \\
(1)\end{array}$ & 6.59 & 99 \\
\hline $\begin{array}{l}\text { Shallow Water } \\
\text { table }\end{array}$ & $\begin{array}{c}0.0 \% \\
(0)\end{array}$ & $\begin{array}{c}2.0 \% \\
(2)\end{array}$ & $\begin{array}{c}0.0 \% \\
(0)\end{array}$ & $\begin{array}{c}6.1 \% \\
(6)\end{array}$ & $\begin{array}{c}24.2 \% \\
(24)\end{array}$ & $\begin{array}{c}37.4 \% \\
(37)\end{array}$ & $\begin{array}{c}15.2 \% \\
(15)\end{array}$ & $\begin{array}{c}5.1 \% \\
(5)\end{array}$ & $\begin{array}{c}8.1 \% \\
(8)\end{array}$ & $\begin{array}{c}2.0 \% \\
(2)\end{array}$ & 6.13 & 99 \\
\hline $\begin{array}{l}\text { Limited Access } \\
\text { to Site }\end{array}$ & $\begin{array}{c}1.0 \% \\
(1)\end{array}$ & $\begin{array}{c}45.5 \% \\
(45)\end{array}$ & $\begin{array}{c}26.3 \% \\
(26) \\
\end{array}$ & $\begin{array}{c}3.0 \% \\
(3)\end{array}$ & $\begin{array}{c}3.0 \% \\
(3) \\
\end{array}$ & $\begin{array}{c}10.1 \% \\
(10)\end{array}$ & $\begin{array}{c}2.0 \% \\
(2)\end{array}$ & $\begin{array}{c}5.1 \% \\
(5) \\
\end{array}$ & $\begin{array}{c}2.0 \% \\
(2)\end{array}$ & $\begin{array}{c}2.0 \% \\
(2)\end{array}$ & 3.52 & 99 \\
\hline $\begin{array}{l}\text { Availability of } \\
\text { Services }\end{array}$ & \begin{tabular}{|c|}
$6.1 \%$ \\
$(6)$ \\
\end{tabular} & $\begin{array}{c}1.0 \% \\
(1)\end{array}$ & $\begin{array}{c}44.4 \% \\
(44) \\
\end{array}$ & $\begin{array}{c}23.2 \% \\
(23)\end{array}$ & \begin{tabular}{|c|}
$7.1 \%$ \\
$(7)$ \\
\end{tabular} & $\begin{array}{c}2.0 \% \\
(2)\end{array}$ & $\begin{array}{c}6.1 \% \\
(6) \\
\end{array}$ & $\begin{array}{c}4.0 \% \\
(4) \\
\end{array}$ & $\begin{array}{c}4.0 \% \\
(4) \\
\end{array}$ & $\begin{array}{c}2.0 \% \\
(2) \\
\end{array}$ & 4.13 & 99 \\
\hline $\begin{array}{l}\text { Effect of Social } \\
\text { Factors }\end{array}$ & $\begin{array}{c}2.0 \% \\
(2)\end{array}$ & $\begin{array}{c}2.0 \% \\
(2)\end{array}$ & $\begin{array}{c}2.0 \% \\
(2)\end{array}$ & $\begin{array}{c}2.0 \% \\
(2)\end{array}$ & $\begin{array}{c}4.0 \% \\
(4)\end{array}$ & $\begin{array}{c}6.1 \% \\
(6)\end{array}$ & $\begin{array}{c}5.1 \% \\
(5)\end{array}$ & $\begin{array}{c}14.1 \% \\
(14)\end{array}$ & $\begin{array}{c}56.6 \% \\
(56)\end{array}$ & $\begin{array}{c}6.1 \% \\
(6)\end{array}$ & 7.95 & 99 \\
\hline
\end{tabular}


Causes of Schedule Overruns in Pakistani Construction Industry

\begin{tabular}{|c|c|c|c|c|c|c|c|c|c|c|c|c|}
\hline $\begin{array}{l}\text { Poor Soil } \\
\text { Conditions }\end{array}$ & \begin{tabular}{|c|}
$0.0 \%$ \\
$(0)$
\end{tabular} & $\begin{array}{c}2.0 \% \\
(2)\end{array}$ & $\begin{array}{c}0.0 \% \\
(0)\end{array}$ & $\begin{array}{c}1.0 \% \\
(1)\end{array}$ & $\begin{array}{c}8.1 \% \\
(8)\end{array}$ & $\begin{array}{c}15.2 \% \\
(15)\end{array}$ & $\begin{array}{c}39.4 \% \\
(39)\end{array}$ & $\begin{array}{c}6.1 \% \\
(6)\end{array}$ & $\begin{array}{c}17.2 \% \\
(17)\end{array}$ & $\begin{array}{c}11.1 \% \\
(11)\end{array}$ & 7.29 & 99 \\
\hline $\begin{array}{l}\text { Safety and } \\
\text { Health }\end{array}$ & \begin{tabular}{|c|}
$62.6 \%$ \\
$(62)$
\end{tabular} & $\begin{array}{c}2.0 \% \\
(2)\end{array}$ & $\begin{array}{c}3.0 \% \\
\text { (3) }\end{array}$ & $\begin{array}{c}2.0 \% \\
(2)\end{array}$ & $\begin{array}{c}2.0 \% \\
(2)\end{array}$ & \begin{tabular}{|c|}
$1.0 \%$ \\
$(1)$
\end{tabular} & \begin{tabular}{|c|}
$7.1 \%$ \\
$(7)$
\end{tabular} & $\begin{array}{c}6.1 \% \\
(6)\end{array}$ & $\begin{array}{c}2.0 \% \\
(2)\end{array}$ & $\begin{array}{c}12.1 \% \\
(12)\end{array}$ & 3.37 & 99 \\
\hline
\end{tabular}

Table-5 Ratings of Causes

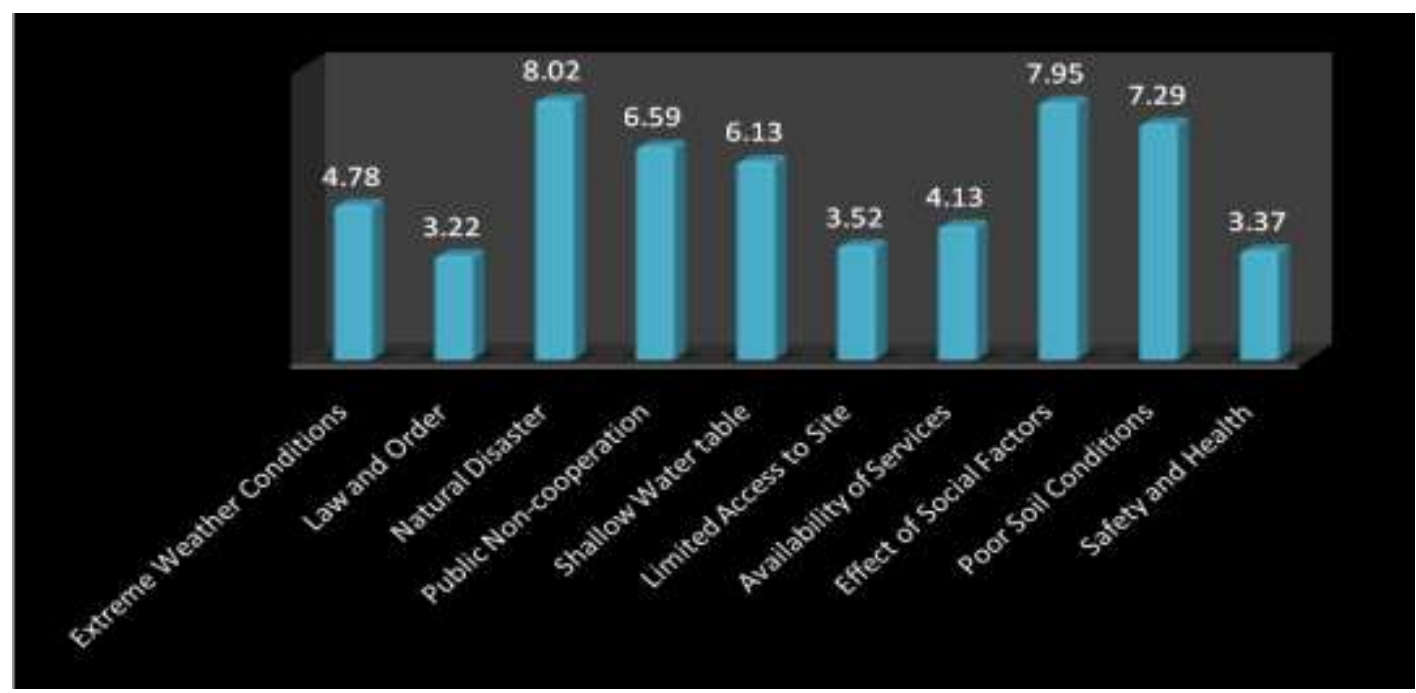

Fig 5 (a): Rankings of Causes

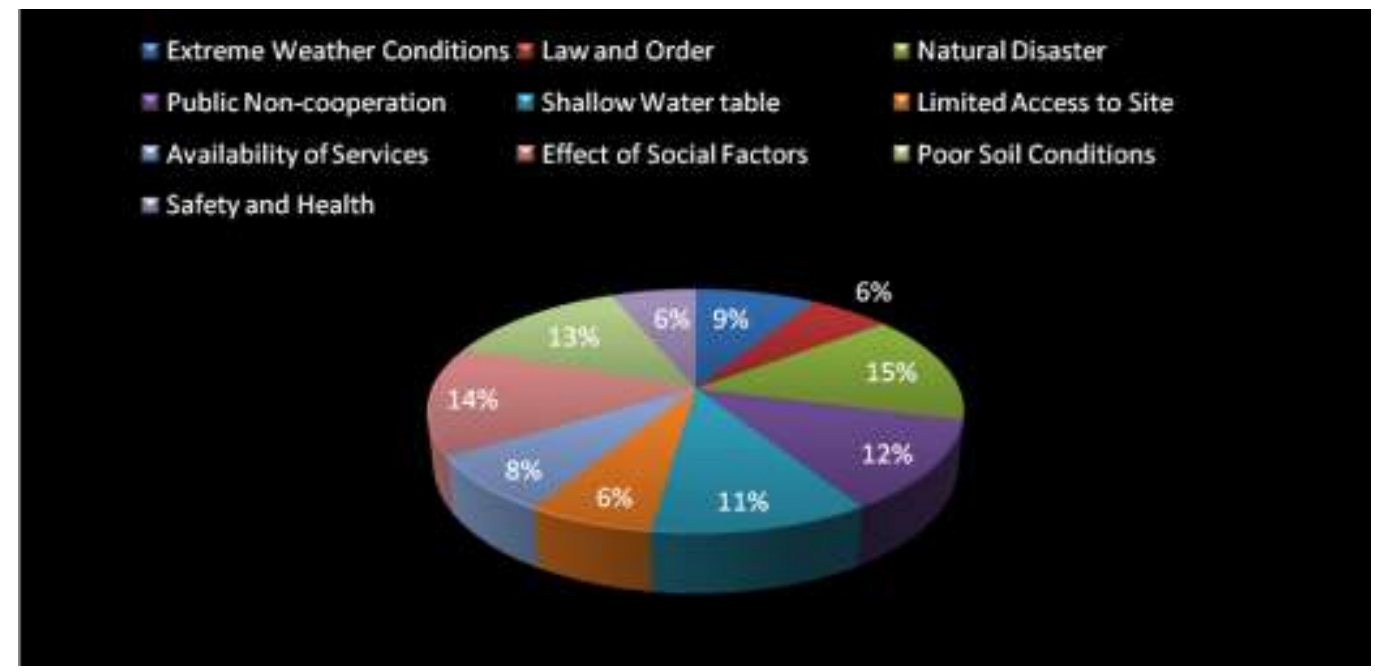

Fig 5 (b): Rankings on pie chart

\section{Findings And Conclusions}

From the results and discussions, following findings and conclusions have been drawn:-

6.1 Paucity of funds and delay in progress payments, severely slow down the projects in terms of time dimension.

6.2 There is a trend of hasty completion of the projects in most of the public service government departments.

6.3 Rapid market fluctuations and hegemony of market players (suppliers) inflict direct negative effects on construction projects.

6.4 Inefficiency and ineffectiveness of site management, results in to reworks, scope creep, scrap, poor quality and frequent change orders by the contractors.

6.5 Planning of judicial use of resources possesses very high significance. Absence or lack of effective planning directly delays the schedule baseline.

6.6 Delay in site mobilization by contractor affects the health of the projects.

6.7 Lack of the modern design software and design data results into design errors in drawings and sub detailing. 
6.8 Delay in approvals of shop drawings and submittals by the consultants, affects the construction schedule of the contractor.

6.9 Lack of Project integration leads to conflicts and constructability issues during execution phase.

6.10 Insufficient funds and frequent changes during execution, delays the project timeline very significantly.

6.11 All stakeholders especially the clients must remain involve during planning phase of the project.

6.12 Timely decision making is a hall mark for the success of a project and vice versa.

6.13 Law and order plays a pivotal role under the global and regional security environments.

6.14 Sometimes limited access to the site becomes problematic in the smooth execution of activities.

\section{RECOMMENDATIONS}

From the findings and conclusions, following viable recommendations have been drawn and suggested in order to avoid unnecessary project delays.

7.1 Availabilities of Sufficient Funds. Majority of the projects get delayed or abandoned due to paucity of funds. The clients must ensure and maintain supply of funds as per cash flow demands. Unnecessary disruptions of cash flows or excessive financial controls hinder the performance and the productivity of the projects.

7.2 Quick Design Approvals. Consultants and clients very often do not offer prompt or timely approvals. Every activity in the construction management plan is harnessed with CPM. Delay in one activity jeopardizes the timelines of succeeding activities. Eventually project slips out of approved schedule baseline. In order to avoid this situation, relevant stakeholders must give timely approval of the drawings, designs or any other submittal.

7.3 Adequate Planning. Lack of detailed planning leads to formation of unrealistic schedule which the contractor cannot follow. Detailed planning eliminates the chances of schedule delays.

7.4 Market Stability. Frequent market fluctuations and price hike by few market players have seriously affected the construction industry. The government should evolve stringent mechanisms and policies to arrest such type of unhealthy trends and unethical practices.

7.5 Site Management and Site Mobilization. Contractors should improve their project management skills and articulate their resources in order to address these two issues for the benefits of their own as well as the projects.

7.6 Project Management Information System. PMIS serves as eyes and ears of any construction project. All efforts in terms of men, machines and materials can be used optimally by using an effective PMIS.

\section{References}

[1] Apolot, 2011, et al, "An investigation into the causes of Delay and Cost Overruns in Uganda's Public Sector Construction Projects".

[2] Baloyi. et al 2011 "Causes of Construction Cost And time Overruns: The 2010 FIFA World Cup Stadia in South Africa", Aeta Structilia 2011.

[3] E.D. Love, et al 2004, A Rework Reduction Model for Construction Projects,", IEEE Transactions on Engineering Management.

[4] Ford, N. David et al 2008, "Project Controls to Minimize Cost and Schedule Overruns: A Model, Research Agenda, and Initial Results.

[5] Haseeb, et al 2011, "Causes and Effects of Delays in Large Construction Projects of Pakistan", Kuwait Chapter of Arabian Journal of Business and Management Review Vol-1, No.4.

[6] Han, Sueng, et al 2009, "Analyzing Schedule Delay of Mega Projects Lessons Learned From Korea Train Express", IEEE Transactions on Engineering Management, Vol.56, No.2.

[7] Hoai, et al 2008, "Delay and Cost Overruns In Vietnam Large Construction Project; A Comparison with other Selected Countries", KSCE Journal of Civil Engineering

[8] Kagapaylan. B, 2008 "Determinants of Project Performance in Russian Construction Industry".

[9] Kasimu M.A, 2012 "Significance Factors that Causes Cost Overruns in Building Construction Project in Nigeria", Interdisciplinary Journal of Contemporary Research in Business.

[10] Lu-Xinhai et al 2001, "Problems of Projects and Effects of Delays in the Construction Industry of Pakistan", Australian Journal of Business and Management Research, Vol 1, NO.5.

[11] Ramanthan, Chidambram, et al 2012, "Analysis of Time and Cost Overruns in Malaysian Construction", Journals of Advanced Materials Research.

[12] Sambasivan Murali, et al 2007, "Causes of and Effects of Delays in Malaysian Construction Industry", International Journal of Project Management.

[13] Shah, Ali, Azlan, et al 2004 A Rework Reduction Model for Construction Projects", IEEE Transactions on Engineering Management.

[14] Vidalis. S.M. et al 2002, "Cost and Time Overruns in Highway Constructions", 4th Transportation Specialty Conference of Canadian Society of Civil Engineering. 